\title{
El sistema de salud del Canadá y su financiamiento ${ }^{1}$
}

\author{
Francisco Xavier Solórzano²
}

RESUMEN El presente trabajo es el fruto de una breve visita realizada en 1993 a los servicios de salud de Canadá como parte del Programa de Formación en Salud Internacional de la OPS, y de un subsiguiente ejercicio de investigación, discusión y análisis. No pretende en modo alguno ser exhaustivo, sino más bien aproximarse a uno de los aspectos relevantes del sistema: $s u$ financiamiento. El objetivo central es identificar algunas de las virtudes y limitaciones de un sistema de salud que se considera de los más eficientes, efectivos y equitativos del mundo.

A pesar de que el gobierno federal y los gobiernos provinciales financian el sistema de salud canadiense, la contención de costos es una preocupación constante, ya que factores como el uso creciente de tecnologías de alta complejidad, la atención hospitalaria y el tratamiento prolongado de las enfermedades crónicas y degenerativas contribuyen a aumentar los costos.

La progresiva reducción del presupuesto federal ha llevado a un uso más eficiente de los recursos y a la racionalización de la capacidad instalada. Asimismo, la relativa sencillez con que funciona el sistema ha permitido mantener bajos los costos administrativos. Además, se han ideado otras formas de atención, como los centros locales de atención comunitaria, la atención domiciliaria y en asilos para promover la autosuficiencia en la medida de lo posible, y el uso de personal voluntario, para controlar parcialmente el incremento de los costos.

La participación de la población en la planificación de los servicios de salud y en la toma de decisiones le permite orientar su desarrollo. Dada la situación actual, resulta imprescindible, sin embargo, modificar el sistema para adecuarlo a los desafíos planteados por el siglo XXI.

En el Canadá la atención de salud se proporciona por un sistema nacional simbiótico cuyo financiamiento es compartido por los seguros de salud público y privado, los usuarios y el gobierno (1). La Constitución canadiense delega la responsabilidad de la atención de salud a los gobiernos provinciales y territoriales. De ahí que existan, en la práctica, 12 sistemas de

Próximamente en esta revista se publicará una versión en inglés de este artículo.

2 Organización Panamericana de la Salud, Programa de Políticas Públicas y Salud, Washington, DC, Estados Unidos de América. Toda correspondencia relacionada con este artículo debe dirigirse al autor a la siguiente dirección postal: P.O. Box 2535, Rockville, MD 20847, EUA. salud entrelazados, correspondientes a 10 provincias y dos territorios (Yukón y Noroeste), más uno bajo jurisdicción federal. Las provincias tienen la responsabilidad primordial de administrar, organizar y ofrecer los servicios y de financiar y regular las actividades de los profesionales de la salud, mientras que el gobierno federal establece el marco legal y los principios básicos del sistema, que las provincias y territorios observan con relativa flexibilidad $(1,2,3)$. El sistema federal también cubre a grupos especiales, como los aborígenes, las fuerzas armadas y la policía federal (4). A pesar de que los sistemas provinciales y el sistema federal son independientes y autóno- mos, todos tienen que cumplir con los principios básicos de accesibilidad, cobertura integral, universalidad, portabilidad y administración pública (5).

El financiamiento es compartido por el gobierno federal y los gobiernos provinciales o territoriales. La legislación federal ha sido diseñada con el fin de garantizar que todos los residentes del país tengan acceso a la atención médica que necesiten mediante un sistema de seguro de salud financiado por impuestos. Independientemente de su ingreso o capacidad de pago, todo residente puede, en principio, recibir servicios médicos y de hospitalización, además de los procedimientos diagnósticos, quirúrgicos y dentales (6) que 
se consideren necesarios desde el punto de vista médico, dentro de la cobertura que ofrece el sistema. A discreción de las provincias y, más aún, de los gobiernos locales, existen ciertos planes de seguro de salud que ofrecen cobertura adicional. Sin embargo, un mandato constitucional impide que se le niegue atención a un residente del país, aunque no posea cobertura o sus recursos económicos no sean suficientes para cubrir el costo de la atención. De hecho, la condición de residente en otro país nunca puede usarse como justificación para negarle la atención a una persona.

Aunque la población canadiense es relativamente pequeña en relación con el tamaño del territorio nacional, tiende a concentrarse en las áreas urbanas, principalmente del sur y el litoral, y la densidad poblacional en las áreas rurales es baja.

\section{MAGNITUD DE LOS GASTOS}

En Canadá, el gasto per cápita en salud y el porcentaje del producto interno bruto (PIB) dedicado a ella figuran entre los más altos del mundo. De hecho, en 1992 el gasto en salud de Canadá se estimó en 9,5\% del PIB, mientras que en Estados Unidos se estimó en 13\% (7). Si estos valores se traducen a gastos per cápita, en 1991 Canadá gastó, por persona, \$US 2 107, mientras que Estados Unidos gastó \$US 2868 (cuadro 1). Cuando se comparan estos datos con los publicados para 1990 por el Banco Mundial (8), se aprecian ciertas diferencias. No obstante, al compararse estas cifras con las de otros países desarrollados, se confirma que el porcentaje del PIB que Canadá y Estados Unidos dedican a la salud es de los más altos del mundo. Junto con Finlandia, Suecia y Suiza, el gasto per cápita en salud en esos países también figura entre los más elevados (8) y se ha duplicado durante la última década.

En 1990, los gastos totales en salud de Canadá se distribuyeron de la siguiente manera: hospitales, $38 \%$; otras instituciones, $11 \%$; medicamentos y equipos, $16 \%$; médicos, $15 \%$; den-

CUADRO 1. Costo y financiamiento de los servicios de salud del Canadá y los Estados Unidos, 1988 y 1991

\begin{tabular}{|c|c|c|c|}
\hline Indicador & Canadá & Estados Unidos & Año \\
\hline $\begin{array}{l}\text { Gasto en salud } \\
\left(\text { en } \$ \text { US } \times 10^{9}\right)\end{array}$ & 56,9 & 751,8 & 1991 \\
\hline $\begin{array}{l}\text { Gasto en salud } \\
\text { (en \% del PIBa) }\end{array}$ & 9,9 & 13,2 & 1991 \\
\hline $\begin{array}{l}\text { Gasto per cápita } \\
\text { (en \$US) }\end{array}$ & 2107 & 2868 & 1991 \\
\hline \multicolumn{4}{|l|}{ Servicios hospitalarios } \\
\hline $\begin{array}{l}\text { Camas disponibles } \\
\text { (por } 1000 \text { habitantes) }\end{array}$ & 5 & 3,9 & 1988 \\
\hline $\begin{array}{l}\text { Ingresos } \\
\text { (por } 1000 \text { habitantes) }\end{array}$ & 137 & 130 & 1988 \\
\hline $\begin{array}{l}\text { Pacientes operados } \\
\text { (por } 1000 \text { habitantes) }\end{array}$ & 70 & 63 & 1988 \\
\hline $\begin{array}{l}\text { Estancia promedio } \\
\text { (en días) }\end{array}$ & 10,7 & 7,2 & 1988 \\
\hline Tasa de ocupación (\%) & 82 & 66 & 1988 \\
\hline $\begin{array}{l}\text { Costo por estancia } \\
\text { (en \$US) }\end{array}$ & 3660 & 4207 & 1988 \\
\hline $\begin{array}{l}\text { Costo al día } \\
\text { (en \$US) }\end{array}$ & 334 & 744 & 1988 \\
\hline
\end{tabular}

Fuente: Ministerio de Salud del Canadá, 1993. a Producto interno bruto.

tistas, 6\%; actividades de investigación, $1 \%$; funciones administrativas, $1 \%$; y otras categorías, $12 \%$. En Estados Unidos, los gastos se distribuyeron como sigue: hospitales, $38 \%$; funciones administrativas, $6 \%$; medicamentos $\mathrm{y}$ equipos, $10 \%$; médicos, $19 \%$; dentistas, $5 \%$; otras instituciones, $8 \%$; actividades de investigación, $2 \%$; y otras categorías, $12 \%$ (9). Las grandes diferencias en los gastos administrativos de los sistemas canadiense y estadounidense explican, en parte, la brecha entre sus respectivos gastos en salud. De hecho, Estados Unidos gasta en administrar su sistema de salud aproximadamente cuatro veces lo que gasta Canadá $(9,10)$.

\section{EL FINANCIAMIENTO}

La distribución de los gastos en salud de Canadá revela que cerca de 95\% de los que corresponden a médicos y hospitales se cubren con recursos del fisco. Sin embargo, el sector público asume aproximadamente $75 \%$ del gasto total, puesto que la diferencia se financia con fondos directos del bolsillo de los individuos y de seguros privados (11). En los últimos años el gasto privado ha crecido más que el público y como resultado de ello la participación pública se ha reducido a $72 \%$ (1). En realidad, el sector público tiene el papel fundamental de financiar el seguro de salud, en tanto que el privado financia básicamente la mayor parte de los medicamentos, la atención dental y los lentes prescritos $(11,12)$.

El gasto del sector público en salud proviene básicamente de los impuestos y es compartido entre el gobierno provincial $(61 \%)$ y el federal (37\%). La distribución se basa en datos de población y en el PIB per cápita de los últimos 3 años (13). Los gobiernos locales y los fondos de compensación de los trabajadores comparten el $2 \%$ restante. El gasto federal en salud proviene, en su mayor parte, del fondo consolidado de rentas, que incluye, entre otras cosas, aranceles aduaneros e impuestos sobre 
la renta de individuos y compañías, sobre las importaciones y sobre el consumo. En cambio, lo que las provincias gastan en salud proviene principalmente de la recolección general de impuestos. En Ontario, por dar un ejemplo, 33\% proviene de impuestos sobre la renta de las personas; $18 \%$ de impuestos sobre las ventas, y $7 \%$ de impuestos sobre la renta de las compañías. Existen, además, otras contribuciones: el empleador, $6 \%$; el gobierno federal, $16 \%$; y otras fuentes, $20 \%$. Según datos de 1993 proporcionados por el Ministerio de Salud de Canadá, también constituyen pequeñas fuentes complementarias las primas de salud, que representan de 5 a $20 \%$ del gasto en dos provincias; las contribuciones para atención de salud derivadas de los sueldos; y los cobros por el alojamiento y la alimentación de pacientes que requieren una larga hospitalización.

Otra característica interesante del sistema canadiense es que tanto los médicos como los hospitales trabajan independientemente en el sector privado. Como el sistema de salud está basado en el concepto de un pagador único, que en el caso canadiense es el gobierno provincial (con el apoyo del federal), para cada procedimiento medicoquirúrgico se ha establecido una tarifa negociada por común acuerdo entre el gobierno y la asociación médica. Aproximadamente 95\% de los médicos que trabajan en el sistema canadiense reciben un pago por sus servicios y el resto recibe su remuneración en forma de capacitación o de un salario. El establecimiento de tarifas fijas para los distintos servicios profesionales ha creado cierto descontento entre algunos médicos cuyo parámetro de referencia es el ingreso de sus colegas en Estados Unidos. Sin embargo, los médicos son los profesionales mejor pagados de Canadá (14). Solamente 1\% - los que se ocupan fundamentalmente de actividades de salud pública, promoción de la salud y salud comunitaria- dependen del gobierno local y reciben un salario fijo. Como resultado del descontento generado por el uso de un sistema de tarifas, se observa una tendencia entre los médicos a solicitar consultas y procedimientos medico- quirúrgicos injustificados (6) con el fin de incrementar sus ingresos.

Actualmente se están creando algunos sistemas para controlar los costos, entre ellos el pago de tarifas más bajas por servicios profesionales a médicos con ingresos anuales que superan cierta cantidad. A los médicos que ganen más de \$Can 400000 se les descuenta un tercio del ingreso y a los que ganan más de \$Can 450000 se les deducen dos tercios. Asimismo, para especialistas se han establecido tarifas más bajas, equivalentes a las que cobra un médico general, en aquellos casos en que sus pacientes no han sido remitidos desde el nivel primario (15).

\section{LA ESTRUCTURA DE LOS SERVICIOS DE SALUD}

Los hospitales, y en particular los que prestan atención a enfermos con afecciones agudas, son públicos en tanto que dependen de contratos con el Estado y que sus administradores responden parcialmente al Ministerio de Salud. Sin embargo, los hospitales, por su origen, son en realidad corporaciones privadas sin fines de lucro cuyos directores son administradores profesionales que responden ante una junta directiva con representantes de la comunidad. Cada hospital recibe de la provincia una asignación anual de fondos en forma de un paquete global basado en sus gastos previos. Esto permite a los administradores tener cierta flexibilidad en el manejo de los recursos. Los presupuestos se incrementan anualmente de acuerdo con la situación fiscal del gobierno y para responder a situaciones en que los servicios hospitalarios necesiten una adecuación. En algunas ocasiones es posible conseguir financiamiento suplementario para programas especializados. Asimismo, algunas provincias ofrecen incentivos a los hospitales que adoptan mecanismos de control de costos. Uno de ellos es el programa de ahorro y retención, que permite reasignar los recursos ahorrados como fruto de una gestión eficiente a otras áreas de operación del hospital, en vez de revertirlos al tesoro provincial (13).
El desarrollo de programas especializados ha permitido al gobierno utilizar mecanismos financieros para promover la regionalización y racionalización de algunos servicios, tales como la cirugía a corazón abierto, el transplante de órganos y la resonancia magnética nuclear. Incluso las inversiones de capital en tecnologías ultramodernas, como la tomografía axial computadorizada y la resonancia magnética nuclear, provienen de fuentes privadas y se autorizan solo cuando se ha excedido la capacidad instalada de los servicios disponibles. Pero fuera de este tipo de servicios de alta complejidad y de alto costo, que requieren una racionalización del gasto gubernamental, la coordinación interinstitucional es mínima y pocas veces se rinden las debidas cuentas a los organismos de planificación municipales o regionales.

Aunque hay hospitales especializados en el área de la salud mental, los gobiernos provinciales también tienen hospitales psiquiátricos. Actualmente se promueve un sistema de hospitales que abren durante el día y se está disminuyendo el número de camas disponibles, con la finalidad de aumentar la efectividad de la atención psiquiátrica en función de su costo.

Los hospitales no tienen la autorización para desarrollar su actividad con fines de lucro y se ven obligados a idear otras estrategias para financiar sus presupuestos. Entre ellas figuran la utilización de un gran número de voluntarios para las actividades administrativas y de servicio comunitario y la recolección de fondos mediante campañas publicitarias, donaciones y la venta de servicios auxiliares de diagnóstico, tratamiento y rehabilitación a otras instituciones que carecen de la capacidad instalada necesaria para ofrecerlos. El levantamiento de fondos se ha convertido, de hecho, en una actividad particular y especializada con su propio ámbito de acción en el contexto de la organización hospitalaria. En todo caso, es importante reconocer que una de las virtudes del sistema es la relativa armonía entre lo público y lo privado: los profesionales ejercen con relativa libertad, los hospitales son administrados por grupos 
privados que representan a la comunidad a la que sirven, y el Estado financia el quehacer de los hospitales y de los profesionales.

\section{EL CONTROL DE LOS COSTOS}

Durante los últimos años, el presupuesto que asigna el gobierno provincial a los hospitales ha disminuido progresivamente en términos absolutos, lo cual obliga a estas instituciones a ser cada vez más eficientes en el uso de sus recursos y a racionalizar la utilización de su capacidad instalada. Para controlar los costos, los hospitales están asignando sus equipos de salud a actividades de atención comunitaria. En otras palabras, la atención es domiciliaria siempre que se justifique, a fin de evitar que pacientes con trastornos poco peligrosos congestionen la sala de emergencias de los hospitales. La definición clara de sus ámbitos de especialidad es otra estrategia empleada por los hospitales para lograr la remisión adecuada de los pacientes y la compra y venta de servicios complementarios. Por ejemplo, a pesar de que el sector privado puede instalar y vender servicios de diagnóstico por imágenes, en octubre de 1995 el gobierno federal adquirió la capacidad de regular esta oferta mediante la reducción de su contribución al presupuesto provincial (1). También se han puesto en marcha iniciativas destinadas a ofrecer atención a los ancianos en asilos y en su propio hogar. Aunque estas iniciativas dependen en gran parte del apoyo de personal voluntario, el alojamiento y la alimentación, sobre todo en los asilos, tienen un costo. Este se establece de tal forma, sin embargo, que aun los ancianos que dependen de la pensión como única fuente de ingresos puedan pagar su estancia y alimentación y disponer de un mínimo de $\$$ Can 50,00 para gastos de bolsillo. El problema radica más bien en la escasez de camas y en las largas lista de espera.

También se han hecho propuestas metodológicas para racionalizar el uso de los recursos y planificarlos de forma más efectiva. La provincia de Ontario, por ejemplo, ha adoptado un enfoque para la asignación de recursos y la planificación de la atención de salud basado en las necesidades de la población (16). En medio de los cambios que se están operando en el sistema para tratar de controlar los costos crecientes, existen enormes diferencias entre este sistema y los de América Latina, sobre todo en la disponibilidad de recursos financieros para el funcionamiento de los servicios de salud. Como ejemplo de esta brecha basta señalar que, en 1992, el presupuesto de operación del hospital North York en Toronto, que posee alrededor de 500 camas y cuya población de usuarios es de aproximadamente 250000 habitantes, fue de \$Can 94 millones, presupuesto comparable al de algunos ministerios de salud de países en desarrollo, como Nicaragua o Paraguay, con una población 20 veces mayor $(17,18)$.

Canadá también está sufriendo una crisis económica que se ha manifestado en el sector de la salud, principalmente en forma de una reducción de la contribución federal para el financiamiento del seguro de salud. Esto ha obligado a las provincias a recurrir a otras opciones de financiamiento, como la racionalización de la utilización de los servicios de alta complejidad, la promoción de estrategias de atención primaria, y la implementación y fortalecimiento de servicios comunitarios de atención de salud. De hecho, Quebec ha organizado una red de atención a través de los Centros Locales de Servicios Comunitarios (CLSC), que son centros para la atención integral y multidisciplinaria de los problemas sociales de la comunidad, como la drogadicción, el desempleo y la atención del sida y otras enfermedades. Estas unidades de atención, que disponen de poco personal y recursos, basan su operación en la demanda. No obstante, el trabajo conjunto en equipo que desarrollan los profesionales de los CLSC ofrece un ejemplo interesante. Aunque la experiencia de Quebec en este sentido es la más extensa hasta ahora, otras provincias están tratando de emularla.

En la actualidad, los sistemas de salud de Canadá y Estados Unidos enfrentan una crisis debido fundamentalmente al incremento de los costos de los servicios. En 1994 Canadá introdujo una serie de medidas de contención de costos que han generado insatisfacción y malestar entre el personal de salud y los pacientes. Estas medidas incluyen, entre otras, la reducción del número de camas de hospital; el fomento de la cirugía de estancia corta; el incremento de los servicios ambulatorios; el control de la remuneración del personal de salud; el uso más intensivo de otros profesionales; la planificación de especialidades médicas por territorios, y el incentivo a la práctica en áreas rurales $(6,19)$.

El reto que enfrenta Canadá en años venideros consiste, por consiguiente, en poder ofrecer a su población una atención de salud efectiva, equitativa y lo más barata posible. Cabe señalar que la sencillez administrativa del sistema de salud de Canadá ha contribuido a mantener bajos los costos de su administración, con lo que se ha logrado un buen ahorro de recursos. El sistema a base de un pagador único también ha representado un esfuerzo por contener los costos mediante el control de la cantidad de servicios y su precio (7). Con este fin se está promoviendo el uso de métodos baratos de diagnóstico y tratamiento. Por ejemplo, los formularios provinciales restringen el uso de medicamentos caros cuando existen opciones más baratas. No obstante, esta interesante mezcla entre lo público y lo privado, representada por un seguro de salud relativamente barato y financiado por el Estado pero dependiente de proveedores privados, ha dado como resultado el incremento del gasto y de la utilización de los servicios per cápita (20).

\section{CONCLUSIONES}

En Canadá, el Estado garantiza el acceso de sus residentes a los servicios de salud y proporciona la infraestructura y los recursos necesarios para que el sector privado los provea. Los usuarios participan activamente en la planificación y en la toma de decisiones 
mediante su representación en las juntas directivas de los servicios de salud.

Una importante iniciativa actual es el control de costos por medio de estrategias de apoyo comunitario. Indudablemente constituye una opción válida frente al incremento de los costos de proveer servicios de salud a una población que envejece paulatinamente, aportando una creciente carga de enfermedades crónicas y degenerativas que exigen procedimientos de alta complejidad, largos y costosos. Entre las opciones adoptadas figura el establecimiento de servicios de apoyo comunitario para reforzar la labor del personal de salud y de los voluntarios.

Con objeto de contener los costos, la mayor parte de las provincias han adoptado la política de congelar el gasto. En el caso de Ontario, el aumento general de los presupuestos hospitalarios se ha mantenido por debajo de la tasa de inflación, o en alrededor de 1\% (5). Además de las medidas orientadas a controlar el crecimiento presupuestario, también se ha llegado a un acuerdo para limitar los salarios y descontar una proporción determinada del ingreso anual de médicos que ganen más del monto acordado.

A pesar de los elevados costos actuales que enfrenta el sistema de salud canadiense, la calidad de los servicios, la alta satisfacción de los consumidores $y$, sobre todo, el nivel de desarrollo humano alcanzado por la población parecen justificar plenamente la magnitud del gasto en salud. Sin embargo, el dilema actual creado por las reducciones presupuestarias radica en cómo ahorrar recursos sin afectar a la cantidad y calidad de la atención de salud.

En resumen, durante las dos últimas décadas Canadá ha logrado mantener un equilibrio relativamente bueno entre el modelo de atención de salud libre y empresarial de Estados Unidos y los sistemas únicos e integrales, como el británico o el sueco, en que el Estado ejerce un control más riguroso. El sistema canadiense está orientado al bienestar social, es decir, a satisfacer las necesidades básicas de la mayor parte de la población, una de las cuales es precisamente la salud (21). Si se sopesan sus virtudes y deficiencias, el sistema canadiense parece superior al de Estados Unidos. La diferencia radica en los fundamentos de cada sistema: mientras que el sistema estadounidense defiende a ultranza la libertad individual, el canadiense se preocupa por el bienestar colectivo. Uno de los grandes problemas del momento es el continuo incremento del gasto en salud, cuyo control se ve limitado por el envejecimiento de la población, unido a la creciente necesidad de atención hospitalaria, y el enorme consumo de nuevas tecnologías.

La presente década es un momento crucial en que urge corregir algunos de los problemas del sistema de salud de Canadá, para evitar un deterioro progresivo de la accesibilidad y calidad de la atención y prevenir mayores incrementos de los costos. Con estos fines se han propuesto diversas reformas: el desarrollo de actividades de prevención y promoción de la salud que complementen los servicios curativos; la contención de costos y la reducción del déficit gubernamental; la completa racionalización, integración, regionalización y reestructuración de los servicios de salud, con mayor énfasis en su eficiencia y eficacia y en formas alternas de prestar servicios; la descentralización de las responsabilidades; la adopción y uso cuidadosos de innovaciones tecnológicas, reparando en su efectividad en función del costo; y finalmente, la atención a las necesidades de la población, aprovechando toda oportunidad de reducir el riesgo y la carga de morbilidad. ${ }^{3}$

Agradecimiento. Se agradecen profundamente los comentarios y sugerencias de todas las personas que han ayudado a revisar las versiones preliminares de este manuscrito $y$, sobre todo, las observaciones de los tres revisores anónimos que orientaron esta versión final. Cualquier error u omisión es la entera responsabilidad del autor.

\footnotetext{
3 Desde la observación inicial del sistema de salud canadiense y la preparación del presente manuscrito, el sistema se ha visto afectado por una serie de cambios políticos y económicos. Actualmente se advierte una marcada tendencia hacia la reducción del aporte gubernamental para el financiamiento del sistema, lo que ha aumentado la participación del sector privado. Esta situación está generando un conflicto entre las disposiciones legislativas de orden federal y su aplicación en el nivel provincial. El sistema de salud canadiense ha pasado, indudablemente, por un proceso de adaptación constante en un esfuerzo por satisfacer las necesidades de la población. Sin embargo, en este momento es difícil predecir el futuro de un sistema de salud que, pese a sus limitaciones, constituye un modelo para muchos países.
}

\section{REFERENCIAS}

1. National Forum on Health. The public and private financing of Canada's health system. Ottawa: National Forum on Health; 1995.

2. Ministry of Supply and Services. Choose Canada: for world class health products and services. Ottawa: Ministry of Supply and Services; 1992.

3. Health Canada. Canadian report to the Special Meeting on Health Sector Reform. Informe presentado en la Reunión Especial sobre la Reforma del Sector de la Salud, Washington, DC, 29 a 30 de septiembre de 1995.

4. Sutherland R, Fulton J. Spending smarter and spending less: policies and partnerships for health care in Canada. Ottawa: The Health Group; 1994.

5. Fulton J. Canada's health system: bordering on the possible. Washington, DC: Faulkner \& Gray's Healthcare Information Center; 1993.
6. Heidemann E. The Canadian health care system: cost and quality. Bull Pan Am Health Organ 1994; 28:169-176.

7. Chernomas R, Sepehri A. An economist's brief guide to the recent debate on the Canadian health care system. Int J Health Serv 1994;24: 189-200.

8. Banco Mundial. Informe sobre el desarrollo mundial 1993. Washington, DC: BM; 1993. 
9. Himmelstein DM, Woolhandler S. Cost without benefit: Administrative waste in U.S. health care. N Engl J Med 1986;314:441-445.

10. Evans RG, Lomas J, Barer ML, et al. Controlling health expenditures: the Canadian reality. N Engl J Med 1989; 320:571-577.

11. Naylor D. The Canadian health care system: a model for America to emulate? Health Econ 1992;1:19-37.

12. Evans R. Health care in the Canadian community. En: Bennett A, Adams O. Looking north for health: what we can learn from Canada's health care system. San Francisco: Jossey-Bass Publishers; 1993.

13. Díaz-Muñoz R, Levcovich M, Lima Quintana L, Ramos S. Sistemas de salud en proceso de reforma. Buenos Aires: Organización Panamericana de la Salud; 1994.

14. Graig LA. Health of nations. Washington, DC: Congressional Quarterly, Inc.; 1993.
15. United States General Accounting Office. Primary care physicians: managing supply in Canada, Germany, Sweden, and the United States. Informe presentado ante el Presidente del Comité de Operaciones Gubernamentales, Cámara de Representantes de la Oficina de Contabilidad de los Estados Unidos de América, Washington DC, mayo de 1994.

16. Roemer MI. National health systems throughout the world. Ann Rev Public Health 1993; 14:335-353.

17. North York General Hospital. Meeting the challenge: North York General Hospital annual report 1991-92. North York: North York General Hospital; 1992.

18. Organización Panamericana de la Salud. II, Las condiciones de salud en las Américas. Washington, DC: OPS; 1994. (Publicación científica 549).

19. Contandriopoulos AP, Champagne F, Denis J, et al. Regulatory mechanisms in health care sys- tems of Canada and other industrialized countries: Description and assessment. Ottawa: University of Ottawa; 1994.

20. Newbold KB, Eyles J, Birch S. Equity in health care: Methodological contributions to the analysis of hospital utilization within Canada. Soc Sci Med 1995;40:1181-1192.

21. Eyles J, Birch S. A population needs-based approach to health-care resource allocation and planning in Ontario: a link between policy goals and practice? Can J Public Health 1993;84:112-117.

Manuscrito recibido el 6 de septiembre de 1995 y aceptado para publicación en versión revisada el 11 de junio de 1996

ABSTRACT This work stems from a brief visit in 1993 to the Canadian health services as part of the PAHO International Health Training Program and the subsequent research, discussion, and analysis relating to that experience. By no means is this paper an exhaustive account of the system, but rather a close look at one of its aspects: financing. The main objective is to identify some of the virtues and limitations of a health system that is considered one of the most efficient, effective, and equitable in the world.

Although the Canadian health system is financed by the federal government and the provincial governments, cost containment is a constant concern, since factors such as the growing use of highly complex technologies, hospital care, and long-term treatment of chronic and degenerative illnesses tend to increase costs.

The progressive reduction in the federal budget has lead to more efficient use of resources and the rationalization of installed capacity. At the same time, the relative simplicity of the system's operation has permitted administrative costs to be kept low. In addition, alternative forms of care, such as local centers for community-based care, care at home and in special institutions to promote the maximum level of selfsufficiency, and the use of volunteers, have been devised in order to partially control cost increases.

The people's participation in planning and decision-making permit them to guide the development of the health services. Nevertheless, given the current situation, it is essential that the system be modified to prepare it for the challenges the twenty-first century will bring. 